\title{
A Dynamic Inventory Control Policy Under Demand, Yield and Lead Time Uncertainties*
}

\author{
Mohamed Zied Babaï, Yves Dallery \\ Laboratoire Génie Industriel, Ecole Centrale Paris, France \\ (babai@1gi.ecp.fr, dallery@lgi.ecp.fr)
}

\begin{abstract}
In this paper, we analyze a single-stage and single-item inventory control system with non-stationary demand and uncertain system parameters. We propose two extensions of a previous work on the dynamic reorder point policy (the $\left(r_{k}, Q\right)$ policy [2]). In the first extension, we include three types of uncertainties pertaining to the demand uncertainty, the lead time uncertainty, and the yield uncertainty. We study the impact of these uncertainties on the $\left(r_{k}, Q\right)$ policy and we provide an approximation of the optimal parameters of the policy using a sequential approach under a cycle service level constraint. The approximative parameters obtained in this paper are good ones for small values of the variability of uncertainties. In the second extension, we focus on the demand uncertainty. We determine the optimal parameters of the $\left(r_{k}, Q\right)$ policy using a sequential approach under a fill rate service level constraint. In the two extensions, we focus on the safety stock parameter and we propose a method to compute it.
\end{abstract}

Keywords: inventory control, forecasts, cycle service level, fill rate, safety stock, policy parameters

\section{INTRODUCTION}

There is an abundant literature on inventory control policies which extends since the 30's. The most known policies are the reorder point policy, called also as the $(r, Q)$ policy and the order-up-to-level policy, called as the $(S, T)$ policy. Several other alternatives of these policies are developed such as the $(s, S)$ policy, the $(T, r, Q)$ policy and the $(T, r, S)$ policy. Most of the models given in the literature to analyze these policies assumes a stationary demand and a cycle service level. Note that these policies are static, i.e. their parameters are constant over time. For more details on these policies, the reader is referred to [8] and [10]. There is also some literature that studies dynamic inventory control policies based on the investigations of [4] and [6]. However, this literature is interested in optimal inventory control policies that are not easy for implementation.

In earlier papers [1] and [2], we proposed a dynamic reorder point policy for a non stationary demand, namely, the $\left(r_{k}, Q\right)$ policy. The parameters of this policy are determined using a sequential approach which means that the value of the ordering quantity $Q$ is computed ignoring the impact upon it of the reorder point $r_{k}$. Indeed, the ordering quantity is independent of uncertainties, whereas the reorder point takes into account the impact of the uncertainties by the mean of a safety parameter. To determine the safety parameter, a cycle service level approach is used which means that we impose, at each cycle (time period between two successive orders), that the probability of not having a stockout is higher than a target cycle service level. Our proposed policy in these investigations includes only the uncertainty associated with demand.

In real inventory control systems, besides the randomness related to demand forecasts, several other types of randomness may exist. For example, randomness in the replenishment process, randomness related to product quality, randomness due to the unreliability of suppliers, etc. These

*1-4244-0451-7/06/\$20.00 (C)2006 IEEE randomness implies several uncertainties in the system, such as the lead time uncertainty and the yield uncertainty. Therefore, in order to guarantee a better service level, the computation of the parameters of the control policy have to take into account these uncertainties. There is an extensive literature that studies inventory policies by considering the yield uncertainty [3], [5], [7] and [9]. There is also much work that studies inventory systems under the lead time uncertainty [8] and [10]. However, we remark that in these works the impact of each uncertainty on the policy parameters is analyzed separately and most of the results are given for a stationary demand and static policies. This has motivated us to extend our results concerning the $\left(r_{k}, Q\right)$ policy by including yield and lead time uncertainties.

We also remark that most of the work on dynamic inventory control policies considers a cycle service level to compute the parameters of the policies. So, we extend results provided in [2] by considering a fill rate service level which is amongst the most useful measure of service in inventory control systems.

This paper is organized as follows: in Section 2, we describe the system and the assumptions we consider. In Section 3 , we briefly recall the principle and the parameters of the $\left(r_{k}, Q\right)$ policy. We study the impact of uncertainties on the parameters of the policy and we provide the optimal parameters by considering the three uncertainties simultaneously and a cycle service level. In Section 4, we provide the optimal parameters of the $\left(r_{k}, Q\right)$ policy for a fill rate service level. The conclusions are given in Section 5 .

\section{SYSTEM DESCRIPTION AND ASSUMPTIONS}

We consider a single-stage and single-item inventory system with a non-stationary demand. The system is not capacitated and the inventory replenishment requires a leadtime $L$, as represented in Fig. 1 .

We assume that demand is known by means of uncertain forecasts, i.e. forecasts and forecast uncertainties are given 


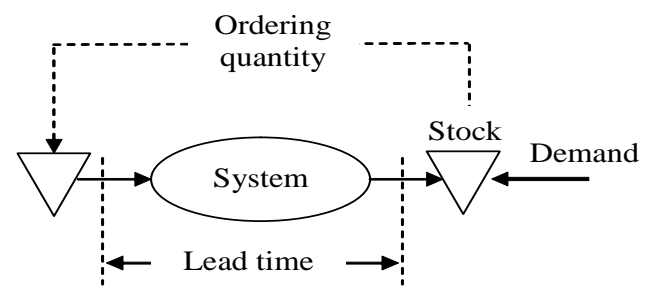

Fig. 1: The inventory system model

for each forecast period. We also assume that Forecast Uncertainties $(F U)$ are independent and identically normally distributed over all the periods of the horizon of forecasts with parameters $\left(0, \sigma_{F U}\right)$. We also consider that the forecast uncertainty is additive, i.e. at each period, the probability distribution of $F U$ is independent of the forecast. For more details on other type of forecast uncertainty models, the reader is referred to [1].

The replenishment lead time $L$ is random. Since, we consider a discrete time control system, we assume that $L$ is a random variable with a discrete probability distribution. The lead time $L$ takes a value $L_{i}$ with a probability $P_{i}$ (i.e. $\left.P\left(L=L_{i}\right)=P_{i}\right)$.

We consider that the supplier is not reliable which implies a yield uncertainty, i.e. if a quantity $Q$ is ordered to replenish the stock, the received quantity $Q_{r}$ is a function of the quantity $Q$ and the yield uncertainty $E Q$, and is expressed as follows: $Q_{r}=Q+E Q$ (i.e. additive uncertainty model). We assume that the yield uncertainty is also normally distributed with parameters $\left(m_{E Q}, \sigma_{E Q}\right)$.

We also consider these notations:

$F_{k}$ : forecast at period $k$

$L$ : replenishment lead time

$I_{k}$ : inventory position at the end of period $k$

$C S L$ : target cycle service level

$A$ : fixed ordering cost

$h$ : holding cost

$H$ : number of periods in the horizon of forecasts

$C F U_{R}$ : cumulative forecast uncertainty over an interval $R$

$\Phi_{C F U_{R}}($.$) : cumulative distribution function of C F U_{R}$

$\phi_{C F U_{R}(.): \text { probability density function of } C F U_{R}}$

$\Phi($.$) : standard normal cumulative distribution function$

$P(x)$ : probability of the random event $x$

\section{THE $\left(r_{k}, Q\right)$ POLICY UNDER DEMAND, YIELD AND LEAD TIME UNCERTAINTIES}

In the $\left(r_{k}, Q\right)$ policy, the system is controlled at each forecast period. At the beginning of each period $k$, if the inventory position falls below the reorder point $r_{k}$, a quantity $Q$ is ordered. The quantity ordered is received after $L$ periods. The inventory level evolution in the $\left(r_{k}, Q\right)$ policy is represented in Fig. 2.

If the lead time $L$ and the order quantity $Q$ are constant, i.e. only the forecasted demand is uncertain, the reorder point $r_{k}$ is equal to the cumulative forecasts over $L+1$ periods plus the safety stock necessary to cover the forecast uncertainty with the cycle service level. The safety stock $S s_{k}$ is equal to the maximal cumulative forecast uncertainty over the protection interval which is equal to $L+1$ periods. The reorder point is given by:

$$
\begin{array}{r}
r_{k}=\sum_{j=1}^{L+1} F_{k+j-1}+S s_{k}^{*} \text { where: } \\
S s_{k}^{*}=\Phi^{-1}(C S L) \sigma_{C F U_{L+1}}
\end{array}
$$

The quantity $Q$ can be computed by using the Wilson's formula, as follows:

$$
Q=\sqrt{\frac{2 A \sum_{i=1}^{H} F_{i}}{h H}}
$$

More details on this policy and their parameters are given in [1] and [2].

In the following, we study the impact of each uncertainty on the $\left(r_{k}, Q\right)$ policy. The optimal parameters under demand, yield and lead time uncertainties are given in Section 3.3 .

\subsection{Impact of the yield uncertainty}

Here, we consider that there is a yield uncertainty in the system, i.e. when a quantity $Q$ is ordered, the received quantity is random. As shown in Fig. 3, a small yield uncertainty does not have any influence on the stockout probability during the replenishment lead time since there is only a shift in the period when the order is placed.

Thus, the equation of the safety stock and the reorder point are as the same as in the $\left(r_{k}, Q\right)$ policy without the yield uncertainty:

$$
r_{k}=\sum_{j=1}^{L+1} F_{k+j-1}+\Phi^{-1}(C S L) \sigma_{C F U_{L+1}}
$$

However, the ordered quantity changes and may be approximated by:

$$
Q=\sqrt{\frac{2 A \sum_{i=1}^{H} F_{i}}{h H}}-m_{E Q}
$$

This approximation is a good one for small values of the variability of the yield. 


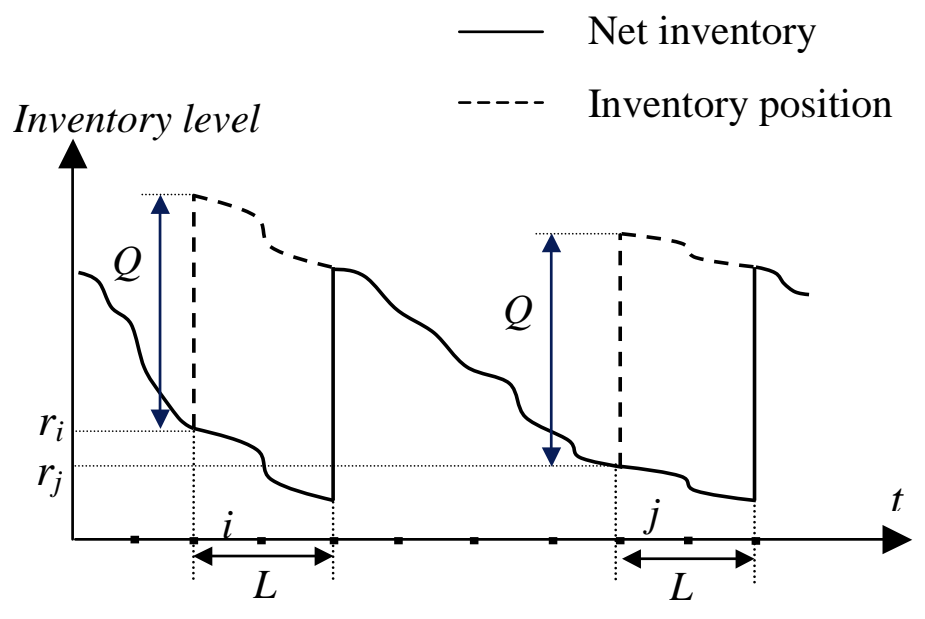

Fig. 2: The $\left(r_{k}, Q\right)$ policy

\subsection{Impact of the lead time uncertainty}

Since we use a sequential approach to determine the parameters of the policy, the optimal ordered quantity is independent of the lead time uncertainty.

However, under the lead-time time uncertainty, at each period $k$ the equation of the reorder point $r_{k}$ changes.

\section{Proposition.}

The reorder point $r_{k}$ can be computed numerically by resolving the equation:

$$
\sum_{i} P_{i} \Phi_{F D_{-} L_{i}+1}\left(r_{k}\right)=C S L
$$

Where $F D_{-} L_{i}+1$ denotes the cumulative forecasted demand over $L_{i}+1$ periods and $\Phi_{F D_{-} L_{i}+1}($.$) its cumulative$ distribution function.

This equation may be solved by using, for example, an algorithm of dichotomy.

\section{Proof.}

The reorder point $r_{k}$ is computed such as:

$$
P\left(\text { Cumulative Demand over } L+1 \leq r_{k}\right)=C S L
$$

Since the lead time $L$ has a discrete probability distribution. By using the Total Probability Theorem and equation (1), we have:

$$
\sum_{i} P_{i} P\left(\text { Cumulative Demand over } L_{i}+1 \leq r_{k}\right)=C S L
$$

Denote by $F D_{-} L_{i}+1$ the cumulative forecasted demand over $L_{i}+1$ periods and by $\Phi_{F D_{-} L_{i}+1}($.$) its cumulative$ distribution function. Hence, at each period $k$ :

$$
\Phi_{F D_{-} L_{i}+1}=C F U_{L_{i}+1}+\sum_{j=1}^{L_{i}+1} F_{k+j-1}
$$

At each period, the random variable $F D_{-} L_{i}+1$ is then normally distributed with a mean $\sum_{j=1}^{L_{i}+1} F_{k+j-1}$ and a standard deviation $\sigma_{C F U_{L_{i}+1}}$ given by:

$$
\sigma_{C F U_{L_{i}+1}}=\sigma_{F U} \sqrt{L_{i}+1}
$$

Thus, the reorder point $r_{k}$ is given by:

$$
\sum_{i} P_{i} \Phi_{F D_{-} L_{i}+1}\left(r_{k}\right)=C S L
$$

Hence, at each period $k$, the reorder point $r_{k}$ can be computed numerically by solving this last equation.

\subsection{Optimal parameters under demand, yield and lead time uncertainties}

The impact of these uncertainties on the $\left(r_{k}, Q\right)$ Policy are studied separately in Section 3.1 and 3.2. In this Section, we give the optimal parameters of the $\left(r_{k}, Q\right)$ policy by considering the demand, yield and lead time uncertainties simultaneously. We assume that uncertainties are relatively small. The result is given by using a sequential approach to satisfy a target cycle service level CSL.

The reorder point $r_{k}$ can be computed numerically by resolving the equation:

$$
\sum_{i} P_{i} \Phi_{F D_{-} L_{i}+1}\left(r_{k}\right)=C S L
$$

The optimal quantity to order is given by: 


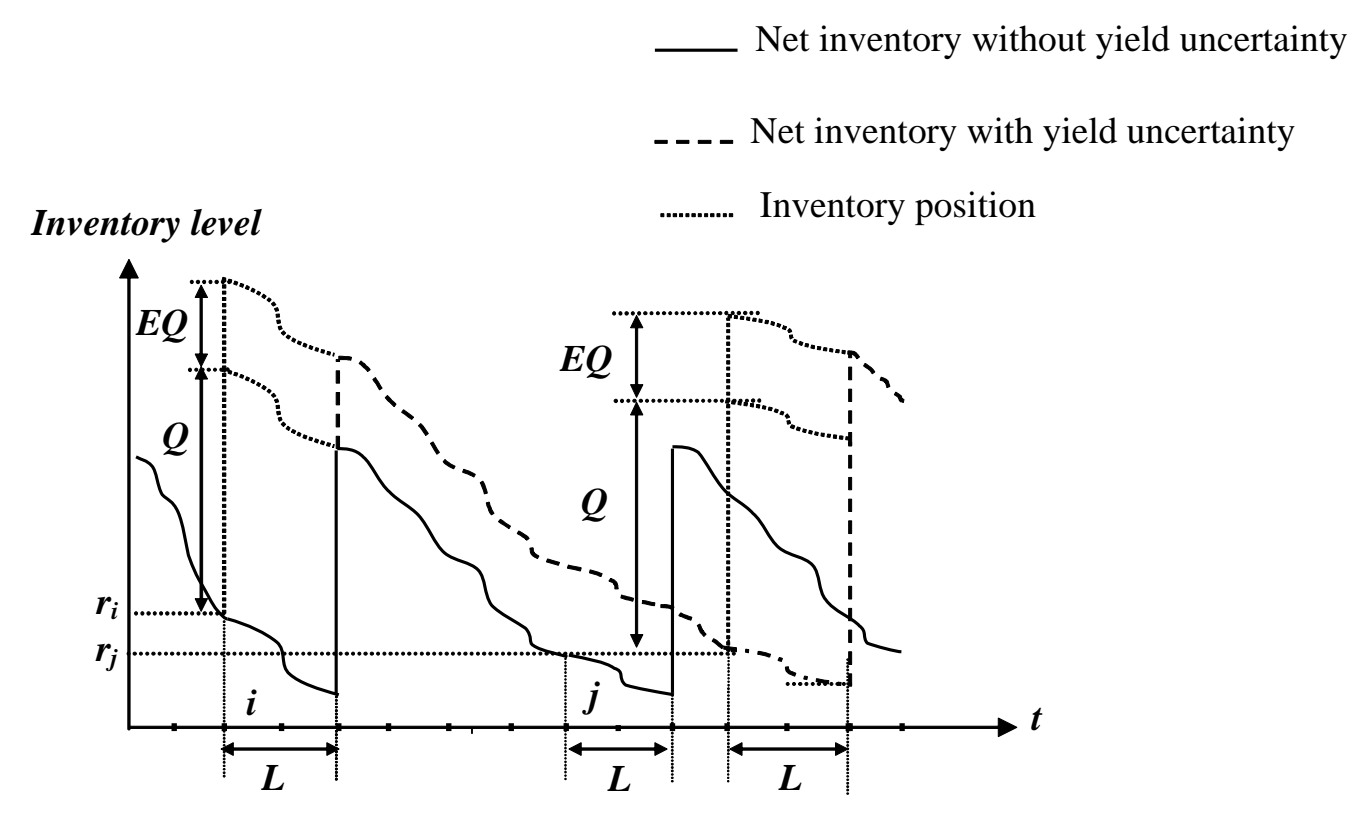

Fig. 3: The $\left(r_{k}, Q\right)$ policy under the yield uncertainty

$$
Q=\sqrt{\frac{2 A \sum_{i=1}^{H} F_{i}}{h H}}-m_{E Q}
$$

\section{THE $\left(r_{k}, Q\right)$ POLICY UNDER A FILL RATE SERVICE LEVEL CONSTRAINT}

In Section 3, a cycle service level approach is used, under three types of uncertainties, to determine the reorder point. In this section, we are interested in a fill rate service level approach, and we consider only a forecast demand uncertainty.

Recall that the cycle service level may be defined as the probability to not have a stockout during a cycle (a cycle is defined as the period between two successive orders). The fill rate is the proportion of demand satisfied directly by the available stock [8].

Let us give a simple example to explain the difference between the cycle service level and the fill rate. We consider the Tab 1. which shows the ordered quantities and the stockout over 10 forecast periods.

In this case, the fraction of periods without stockout is $8 / 10$, thus a cycle service level of $80 \%$ is satisfied. In terms of quantity, only 55 demands are satisfied over a total demand of 1450, so a fill rate of $96.21 \%$ is satisfied ((1450$55) / 1450=96.21 \%$ ).

Since we use a sequential approach, the formula of the optimal quantity to order does not change and is given by:
Tab 1. Example to illustrate service level measures

\begin{tabular}{|c|c|c|}
\hline Period & Demand & Stockout \\
\hline 1 & 180 & 0 \\
\hline 2 & 75 & 0 \\
\hline 3 & 235 & 45 \\
\hline 4 & 140 & 0 \\
\hline 5 & 180 & 0 \\
\hline 6 & 200 & 10 \\
\hline 7 & 150 & 0 \\
\hline 8 & 90 & 0 \\
\hline 9 & 160 & 0 \\
\hline 10 & 40 & 0 \\
\hline Total & 1450 & 55 \\
\hline
\end{tabular}

So, our aim in this section is to compute the reorder point $r_{k}$ necessary to satisfy the target fill rate at each period $k$. Let $\mathrm{Fr}$ denotes the target fill rate, $n\left(r_{k}\right)$ the average number of stockout during a cycle, and $S s_{k}$ the safety stock.

\section{Proposition.}

The optimal safety stock $S s_{k}^{*}$ can be computed numerically by solving the equation:

$$
\begin{aligned}
Q(1-F r)= & -S s_{k}^{*}\left[1-\Phi\left(\frac{S s_{k}^{*}}{\sigma_{C F U_{L+1}}}\right)\right] \\
& +\sigma_{C F U_{L+1}} \phi\left(\frac{S s_{k}^{*}}{\sigma_{C F U_{L+1}}}\right)
\end{aligned}
$$

The optimal reorder point $r_{k}$ is then given by:

$$
r_{k}=\sum_{j=1}^{L+1} F_{k+j-1}+S s_{k}^{*}
$$

$$
Q=\sqrt{\frac{2 A \sum_{i=1}^{H} F_{i}}{h H}}
$$




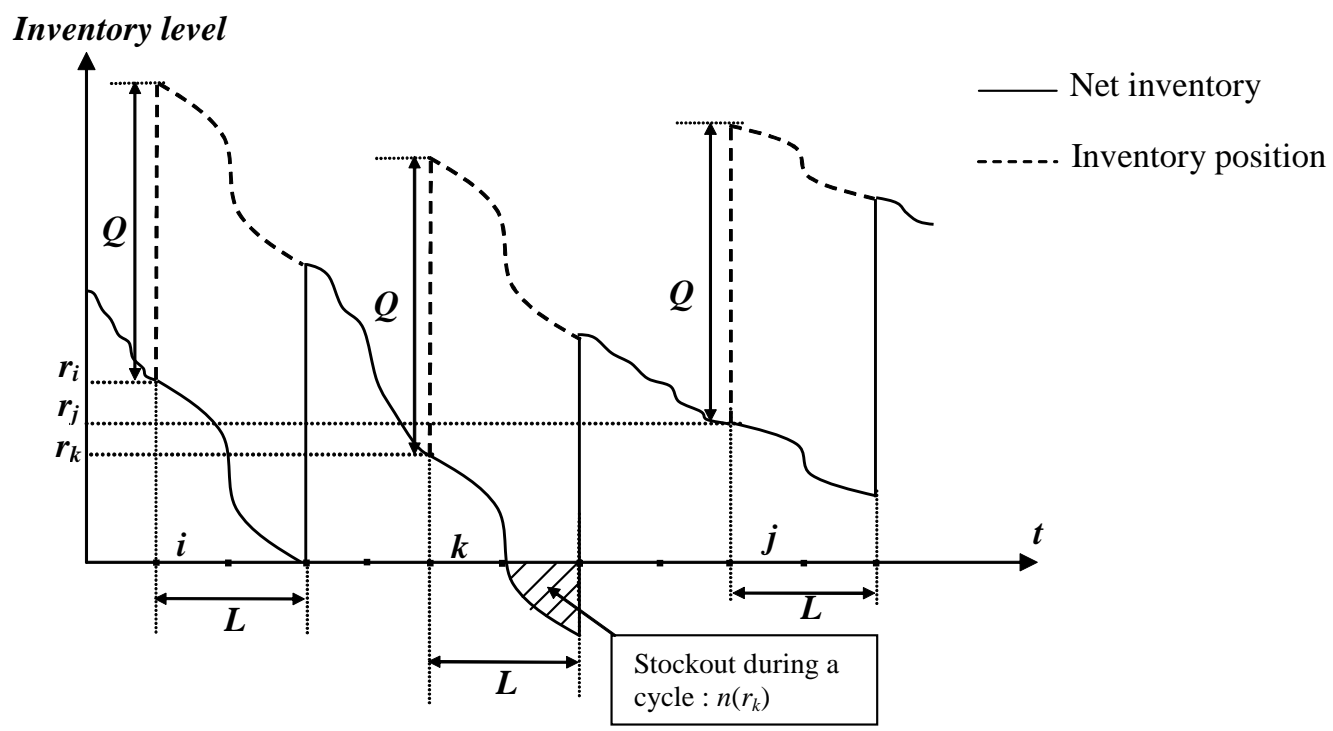

Fig. 4: The $\left(r_{k}, Q\right)$ policy with stockout

\section{Proof.}

At each period $k$, the fill rate is defined as follows:

$$
F r=1-\frac{n\left(r_{k}\right)}{\text { Average demand during a cycle }}
$$

As shown in Fig 4, the demand during the cycle which begins at period $k$ is:

$$
Q+I_{k-1}-I_{j-1}
$$

Where $j$ is the first period where an order is placed after the period $k$.

In practice, if we consider a long horizon of forecasts, we can approximate the average forecasted demand during a cycle by $Q$, where:

$$
Q=\sqrt{\frac{2 A \sum_{i=1}^{H} F_{i}}{h H}}
$$

The average number of stockout, denoted by $n\left(r_{k}\right)$, is defined as follows:

$$
n\left(r_{k}\right)=\int_{x=r_{k}}^{+\infty}\left(x-r_{k}\right) \phi_{L+1}(x) d x
$$

We showed in [1] that:

$$
\begin{aligned}
n\left(r_{k}\right)= & -S s_{k}\left[1-\Phi\left(\frac{S s_{k}}{\sigma_{C F U_{L+1}}}\right)\right] \\
& +\sigma_{C F U_{L+1}} \phi\left(\frac{S s_{k}}{\sigma_{C F U_{L+1}}}\right)
\end{aligned}
$$

Thus, the optimal safety stock $S s_{k}^{*}$ can be computed numerically, by using for example an algorithm of dichotomy, to solve the following equation:

$$
\begin{aligned}
Q(1-F r)= & -S s_{k}^{*}\left[1-\Phi\left(\frac{S s_{k}^{*}}{\sigma_{C F U_{L+1}}}\right)\right] \\
& +\sigma_{C F U_{L+1}} \phi\left(\frac{S s_{k}^{*}}{\sigma_{C F U_{L+1}}}\right)
\end{aligned}
$$

Hence, the reorder point $r_{k}$ is given by:

$$
r_{k}=\sum_{j=1}^{L+1} F_{k+j-1}+S s_{k}^{*}
$$

\section{CONCLUSIONS}

In this paper, we studied the $\left(r_{k}, Q\right)$ policy under demand, yield and lead time uncertainties. A sequential approach is considered and a cycle service level is used to compute the optimal parameters of this policy. We provided good approximations of the optimal parameters of the $\left(r_{k}, Q\right)$ policy for small values of the variability of uncertainties. We showed that, under the yield uncertainty, there is only an impact on the ordered quantity, however, the reorder point does not change. We also showed that, under the lead time uncertainty, the reorder point changes and it can be computed numerically.

In the second part of the paper, we briefly explained the difference between the cycle service level and the fill rate. Then, we determined the optimal parameters of the $\left(r_{k}, Q\right)$ policy using a sequential approach under a fill rate service level constraint. In this part, only the demand uncertainty is considered.

In the future, it would be interesting to develop this analysis by studying the behavior of the optimal parameters for high 
values of the variability of uncertainties. More work could also be done to conduct the same study provided in this paper for other inventory control policies such as the orderup-to-level policies. Another interesting further research consists in developing the optimal parameters of the $\left(r_{k}, Q\right)$ policy for a fill rate service level under demand, yield and lead time uncertainties.

\section{REFERENCES}

[1] M.Z. Babaï, "Politiques de pilotage de flux dans les chaînes logistiques: Impact de l'utilisation des prévisions sur la gestion de stocks", Ph.D. Thesis, Ecole Centrale Paris, 2005.

[2] Babaï, M.Z., Y. Dallery, "An analysis of forecast based reorder point policies: The benefit of using forecasts", Proceedings of the the 12th IFAC Symposium on Information Control Problems in Manufacturing: INCOM'06, Saint-Etienne (France),2006.

[3] Henig, M., Y. Gerchak, "The structure of periodic review policies in the presence of variable yield", $O p$ erations Research, Vol. 38, pp634-643, 1990.

[4] S. Karlin, "Dynamic Inventory Policy With Varying Stochastic Demands", Management Science, Vol. 6, pp231-258, 1960.

[5] Rekik, Y., E. Sahin, Y. Dallery, "A Comprehensive Analysis of the Newsvendor Model with Unreliable Supply", To appear in OR Spectrum, 2005.

[6] H. Scarf, "The optimality of (S,s) policies in the Dynamic Inventory Problem", Mathematical Methods in the Social Sciences, Stanford University Press, Stanford, California, 1959.

[7] E.A. Silver, "Establishing the Reorder Quantity When the Amount Received is Uncertain", INFOR, Vol. 14, pp32-39, 1976.

[8] Silver, E.A., R. Peterson, "Decision Systems for Inventory Management and Production Planning", John Wiley and Sons, New York, 1985.

[9] Yano, C.A., H.L. Lee, "Lot sizing with random yields: a review", Operations Research, Vol. 43, pp311-334, 1995.

[10] P.H. Zipkin, "Foundations of Inventory Management", McGraw-Hill, USA, 2000. 